Please do not remove this page

RMIT

UNIVERSITY

\title{
Study of novel love mode surface acoustic wave filters
}

Kalantar-Zadeh, Koroush; Wlodarski, W; Trinchi, Adrian; Holland, Anthony; Galatsis, Kosmas

https://researchrepository.rmit.edu.au/esploro/outputs/9921857985401341/filesAndLinks?institution=61RMIT_INST\&index=null

Kalantar-Zadeh, K., Wlodarski, W., Trinchi, A., Holland, A., \& Galatsis, K. (2002). Study of novel love mode surface acoustic wave filters. Proceedings of the 2002 IEEE International Frequency Control Symposium and PDA Exhibition, 74-77. https://doi.org/10.1109/FREQ.2002.1075860

Published Version: https://doi.org/10.1109/FREQ.2002.1075860

Repository homepage: https://researchrepository.rmit.edu.au

(c) 2002 IEEE. Personal use of this material is permitted. However, permission to reprint/republish this material for advertising or promotional purposes or for creating new collective works for resale or redistribution to servers or lists, or to reuse any copyrighted component of this work in other works must be obtained from the IEEE.

Downloaded On 2023/04/26 14:05:22 +1000 


\title{
Study of Novel Love Mode Surface Acoustic Wave Filters
}

\author{
Kourosh Kalantar-Zadeh ${ }^{1,2}$, Wojtek Wlodarski ${ }^{1,2}$, Kosmas Galatsis ${ }^{1,2}$, Anthony Holland ${ }^{1}$ \\ 'RMIT University, School of Electrical and Computer Engineering, Melbourne, Australia \\ ${ }^{2} \mathrm{CRC}$ for Microtechnology, Melbourne, Australia
}

\begin{abstract}
Novel Love mode filters based on $\mathrm{ZnO}$ and $\mathrm{SiO}_{2} / 90^{\circ}$ rotated ST-cut quartz crystal structure were fabricated. A comprehensive study was carried out to show the capabilities of such filters. The periodicity of the fingers is $50 \mu \mathrm{m}$ and the thickness of the $\mathrm{SiO}_{2}$ and $\mathrm{ZnO}$ layers ranges from 0.2 to $7.2 \mu \mathrm{m}$. Electromechanical coupling coefficient, capacitance per unit wavelengths of finger pairs and temperature coefficient of frequency are studied in terms of thickness of the wave-guiding layers.
\end{abstract}

Keywords- Filter, SAW, ZnO, Love Wave

\section{I- INTRODUCTION}

Love mode Surface Acoustic Wave (SAW) structures offer significant advantages, making them favourable candidates for the fabrication of high frequency filters. The advantageous features of these structures include: high electromechanical coupling coefficient, low temperature coefficient, small coupling with other wave propagating modes, and high acoustic wave propagation speed.

The crystal cut employed for the fabrication of Love mode filters must allow the propagation of the Surface Skimming Bulk Wave (SSBW) or Leaky SAW [1]. SSBW is a longitudinal bulk wave with Shear Horizontal (SH) polarisation. Furthermore, SSBW has small coupling to other bulk modes of propagation. The crystal with SSBW cut has a lower temperature coefficient than that of Rayleigh crystal cuts. Depositing films of different metal oxides onto such a substrate increases the electromechanical coupling coefficient of the media and can change the mode of operation from SSBW to Love mode. The mode of propagation changes to Love mode when the speed of $\mathrm{SH}$ acoustic wave propagating in the film is less than that of the substrate.

In this paper, novel Love mode SAW based filters will be presented. In these structures, the substrate is $90^{\circ}$ rotated ST-cut quartz crystal and the wave-guiding layers are $\mathrm{SiO}_{2}$ and $\mathrm{ZnO}$.

$\mathrm{SiO}_{2}$ films and $c$-axis oriented $\mathrm{ZnO}$ films were deposited onto the substrate using an r.f. magnetron sputterer. The thicknesses of the deposited films range from 0.1 to $7.2 \mu \mathrm{m}$. Electromechanical coupling coefficients, temperature coefficients of frequency, and capacitance per unit distance of finger pairs for both structures were measured and compared with theoretical calculations.

\section{II- THEORY}

Each port of the SAW filter consists of $\mathrm{N}$ interdigital transducers (IDTs). If $\mathrm{N}$ is large, the input admittance of device is defined as [2]:

$$
G(2 \pi f) \approx 8 N^{2} \mathrm{G}_{0}\left|\frac{\operatorname{Sin}\left(\mathrm{N} \pi\left(f-f_{0}\right) / f_{0}\right)}{\mathrm{N} \pi\left(f-f_{0}\right) / f_{0}}\right|^{2},
$$

where $f_{0}$ is the centre frequency of the filter and $G_{0}$ is the equivalent characteristic admittance of the SAW transmission line. $G_{0}$ is defined as [2]:

$$
G_{0}=K^{2} C_{s} f_{0}
$$

where $K^{2}$ is the electromechanical coupling coefficient and $C_{S}$ is the static capacitance of one periodic section. The remaining equivalent circuit components are two imaginary terms. The first one is the electrodes capacitance [1]:

$$
j B_{c}(2 \pi f)=j 2 \pi f C_{T},
$$

which stands for the admittance generated by the capacitance of the port finger pairs ( $C_{T}$ is the total finger pairs capacitance) and the imaginary part $B_{H}(2 \pi f)$ is the Hilbert transform of equation (1). Hence, the total admittance of one port of a SAW device can be shown to be:

$$
Y(2 \pi f)=G(2 \pi f)+j\left[B_{c}(2 \pi f)+B_{H}(2 \pi f)\right]
$$

The electromechanical coupling coefficient $K^{2}$ is a measure of the efficiency of a given piezoelectric in converting an applied electrical signal into mechanical energy associated with a surface acoustic wave. The experimental equation for $K^{2}$ is [1]:

$$
K^{2}=-2 \frac{|\Delta v|}{v}
$$


where $|\Delta v|$ is the magnitude of the SAW velocity change that occurs when the free surface shorted by a thin, highly conducting metal film.

Capacitance per unit length of finger pairs of a SAW device consisting of two half spaces on the top and bottom of the IDTs is given by the empirical relation [3]:

$$
C_{S}=2\left(\varepsilon_{P R L}^{T}+\varepsilon_{P R}^{T}\right)\left(6.5\left(D_{x} / L_{x}\right)^{2}+1.08\left(D_{x} / L_{x}\right)+2.37\right),(6)
$$

where $D_{x} / L_{x}$ is the finger width to centre-to-centre ratio of two consecutive fingers. The relative equivalent dielectric constant $\varepsilon_{P R}^{T}$ of the substrate is given by $\varepsilon_{P R}^{T}=\frac{\left(\varepsilon_{11} \varepsilon_{33}-\varepsilon_{13}\right)^{1 / 2}}{\varepsilon_{03}}$, where $\varepsilon_{11}, \varepsilon_{33}$, and $\varepsilon_{13}$ are dielectric constants of the substrate at constant stress. $\varepsilon_{P R L}^{T}$ is the relative equivalent dielectric constant of upper half space. Thus the initial and final values for the capacitance can be calculated, using the dielectric constant of air when no layer is deposited $\left(C_{S I}\right)$, and the dielectric constant of deposited film when the thickness of the layer is greater than five wavelengths $\left(C_{S F}\right)$. The capacitance curves for layer thickness $h$, can be fitted better than five percent by [3]:

$$
C=C_{S I}+\left(C_{S F}-C_{S I}\right)\left(1-e^{-4.6 h / L_{x}}\right)
$$

SSBWs in $\mathrm{Y}$ rotated-quartz plates with any rotation angle show a parabolic temperature dependence of frequency with respect to the reference temperature [6]:

$$
\frac{\Delta f}{f_{0}}=T C F_{1}\left(T-T_{0}\right)+T C F_{2}\left(T-T_{0}\right)^{2} .
$$

The magnitude and sign of the temperature coefficients $T C F_{1}$ and $T C F_{2}$ depend on the crystal cut rotation angle. However, Love mode devices show different TCF to that of their substrates.

\section{III- TRANSDUCER FABRICATION}

$\mathrm{ZnO}$ and $\mathrm{SiO}_{2}$ films were deposited on $90^{\circ}$ rotated ST-cut quartz crystal substrates using an r.f. magnetron sputterer [1]. The sputtering conditions are shown in Table I.

\begin{tabular}{|l|l|l|l|l|}
\hline Material & $\begin{array}{l}\text { Spttering } \\
\text { rate }(\mu \mathrm{m} / \mathrm{h})\end{array}$ & $\begin{array}{l}\text { Sputtering } \\
\text { Pressure } \\
\text { (Torr) }\end{array}$ & $\begin{array}{l}\text { Power } \\
(\mathrm{W})\end{array}$ & Gas \\
\hline $\mathrm{SiO}_{2}$ & 0.9 & $1.1 \times 10^{-3}$ & 140 & $\begin{array}{l}\% 90 \mathrm{Ar}, \\
\% 10 \mathrm{O}_{2}\end{array}$ \\
\hline $\mathrm{ZnO}$ & 0.63 & $1.1 \times 10^{-3}$ & 60 & $\begin{array}{l}\% 60 \mathrm{Ar}, \\
\% 40 \mathrm{O}_{2}\end{array}$ \\
\hline
\end{tabular}

Table I. Sputtering conditions.
The velocity of the shear horizontal ( $\mathrm{SH}$ ) acoustic wave in the film should be less than that of the substrate to allow the propagation of Love modes. Depositing $\mathrm{ZnO}$ or $\mathrm{SiO}_{2}$ layers onto ST-cut quartz crystal substrate forms the required condition for Love mode wave propagation. The shear horizontal acoustic wave velocity in $\mathrm{ZnO}$ layer, $\mathrm{SiO}_{2}$ layer and ST-cut quartz substrate are $2650 \mathrm{~m} / \mathrm{s}, 2850 \mathrm{~m} / \mathrm{s}$ and $5060 \mathrm{~m} / \mathrm{s}$, respectively.

The $\mathrm{ZnO}$ films have cylindrical grains grown on initial layer of randomly oriented crystallites. The radius of the $\mathrm{ZnO}$ grains varies between 50 to $200 \mathrm{~nm}$ (Fig. 1).

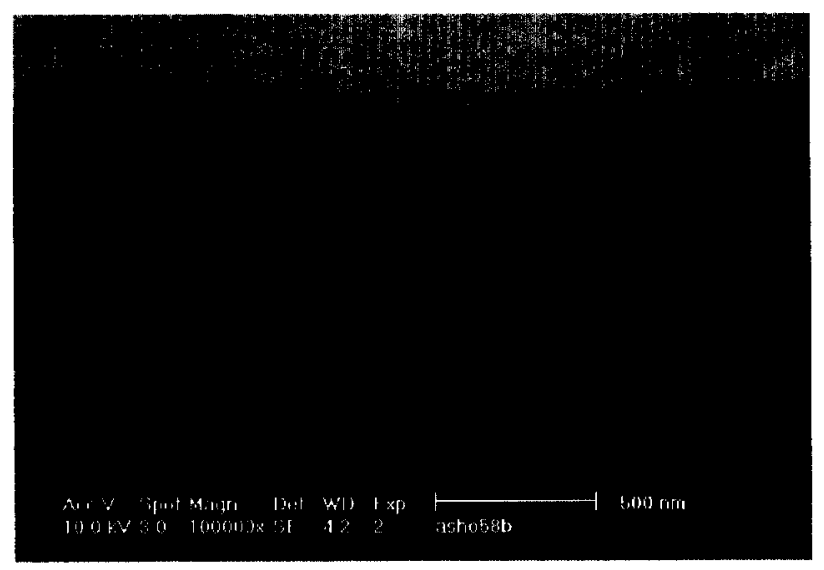

Fig. 1 SEM micrographs of sputtered $\mathrm{ZnO}$.

The IDTs were patterned between the substrate and the waveguiding layers. The IDTs electrode periodicity was $50 \mu \mathrm{m}$. The device consists of 16 IDTs in the input port and 64 IDTs in the output port. Centre-to-centre IDTs distance was 60 wavelengths and aperture was $2.5 \mathrm{~mm}$.

The thickness of the $\mathrm{ZnO}$ and $\mathrm{SiO}_{2}$ layers ranged from 0.2 to $7.2 \mu \mathrm{m}$.

\section{IV-MEASUREMENTS}

Fig. 2 shows the experimental measurements of frequency response of a blank filter with $90^{\circ}$ rotated ST-cut quartz substrate and filters with $\mathrm{SiO}_{2}$ and $\mathrm{ZnO}$ deposited layers. The wavelength of the acoustic waves in the investigated device is $50 \mu \mathrm{m}$. The thickness of $\mathrm{ZnO}$ and $\mathrm{SiO}_{2}$ layers are $1.5 \mu \mathrm{m}$. It is observed that, the $\mathrm{ZnO} / 90^{\circ}$ rotated ST-cut quartz crystal structure has the lowest insertion loss among the other structures. The insertion loss for Love mode device with $\mathrm{ZnO}$ layer is $19 \mathrm{~dB}$ less than that of the blank quartz crystal filter and $6 \mathrm{~dB}$ better than $\mathrm{SiO}_{2} / 90^{\circ}$ rotated ST-cut quartz crystal structure. The acoustic wave propagation modes in both layered structures are predominantly Shear Horizontal (SH). As the propagation velocities in the deposited layers are less than that of the substrates, the propagation modes are transformed to Love mode. Insertion losses are smaller in these layered structures, as SSBWs have changed to Love waves which are mostly propagating near the active surface and in the layers. 
The $\mathrm{ZnO}$ based acoustic device has less insertion loss due to strong piezoelectricity of $\mathrm{ZnO}$, which increases the electromechanical coupling coefficient of the media.

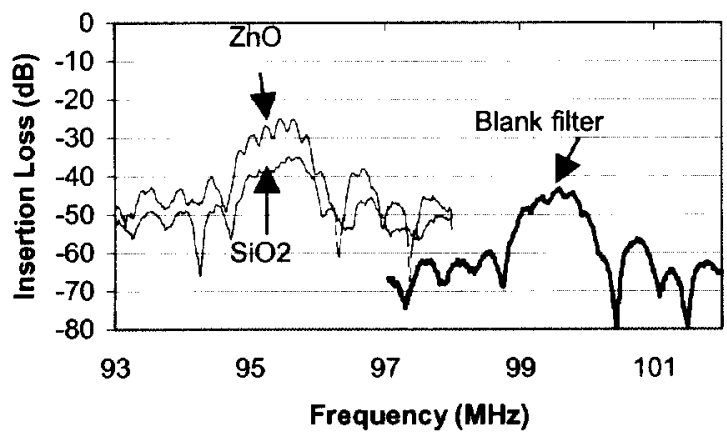

Fig. 2 Frequency response of the ZnO/ST-cut quartz crystal structure, $\mathrm{SiO}_{2} / \mathrm{ST}$-cut quartz crystal structure and blank filters.

Input resistance and capacitance were measured using a network analyser and the electromechanical coupling coefficient and capacitance per unit wavelength of IDT were extracted from these values.

For a blank $90^{\circ}$ rotated ST-cut quartz crystal, $K^{2}$ is 0.017 . Theoretical calculations show that for $\mathrm{SiO}_{2}$ films $K^{2}$ increases and reaches a maximum of 0.028 . For the device with $\mathrm{ZnO}$ film, $K^{2}$ reaches a significantly larger value of 0.06 . $K^{2}$ in both structures has a maximum value at a thickness equal to 0.04 of the wavelength. Theoretical calculations are in excellent agreements with measurements (Fig. 3).
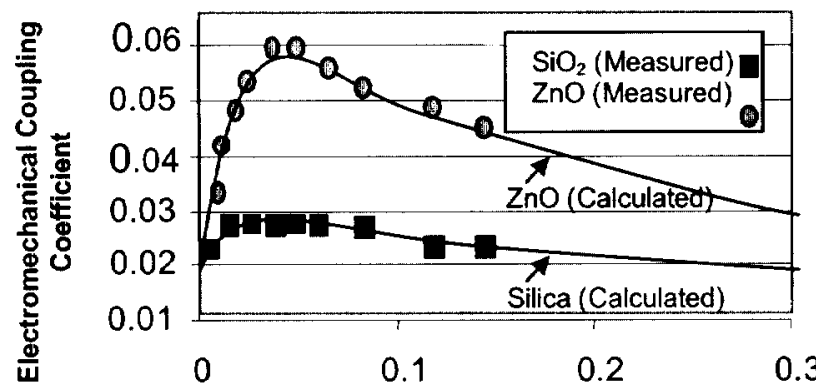

Normalised film thickness (h/wavelength)

Fig. 3 Electromechanical coupling coefficient vs. normalised film thickness.

Equation (7) was used for calculating of the capacitance per unit length. In (7), the relative dielectric constant for $\mathrm{SiO}_{2}$ is 4.4 and for $\mathrm{ZnO}$ is $9.1\left(\frac{\varepsilon_{11}}{\varepsilon_{0}}=8.55, \frac{\varepsilon_{13}}{\varepsilon_{0}}=10.2\right.$ and $\varepsilon_{33}=0$ ). Both measurements results and theoretical values (Fig. 4) are in good agreement.

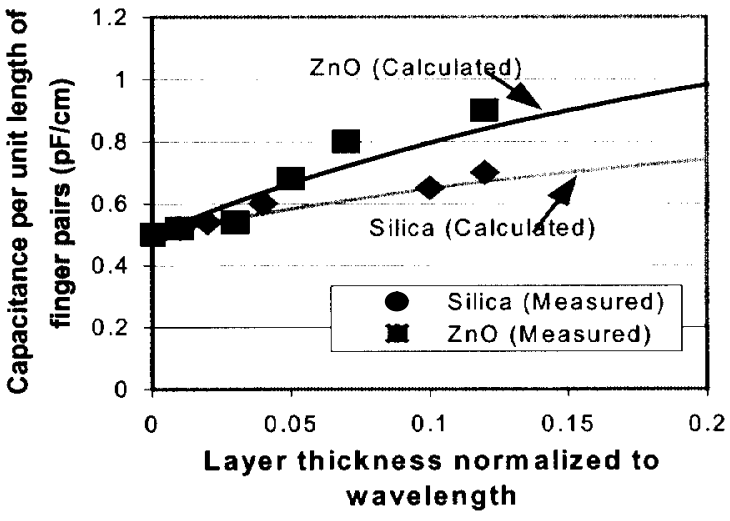

Fig. 4 Capacitance per unit length of IDT finger pairs vs. the normalised layer thickness.

Values of $T C F$ s were measured in an environmental chamber in a temperature range from $-20^{\circ} \mathrm{C}$ to $40^{\circ} \mathrm{C}$. Fig. 5 shows the frequency shift versus temperature change for different $\mathrm{ZnO}$ thicknesses. At low temperatures, it was not possible to calculate the values of $T C F_{2}$. Values of $T C F_{1}$ are determined from the slope of frequency temperature curves in Fig. 5.

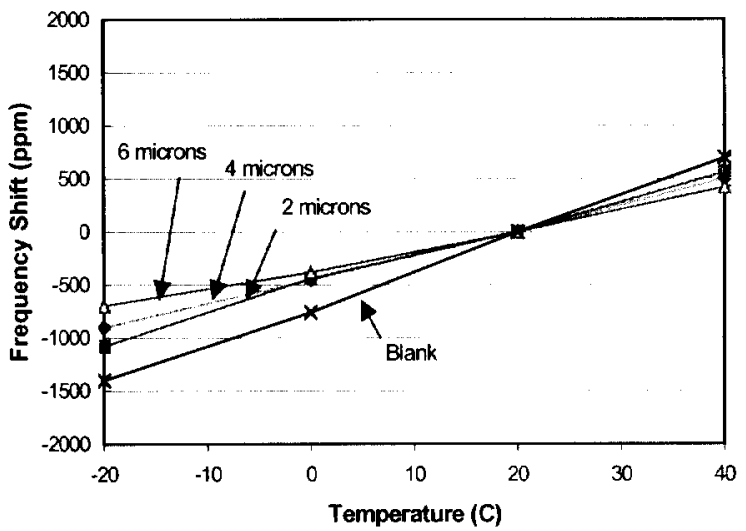

Fig. 5 Frequency shift vs. temperature change at different $\mathrm{ZnO}$ thicknesses.

\section{V-CONCLUSIONS}

In this paper a thorough study of two different structures of Love mode SAW filters was reported. $\mathrm{SiO}_{2}$ and $\mathrm{ZnO}$ were deposited onto $90^{\circ}$ rotated ST-cut quartz crystal. $\mathrm{SiO}_{2}$ films and $\mathrm{c}$-axis oriented $\mathrm{ZnO}$ films were deposited on the substrate using an r.f. magnetron sputterer. Thicknesses of deposited films range from 0.1 to $7.2 \mu \mathrm{m}$. Electromechanical coupling coefficients of frequency, temperature coefficients, and capacitance per unit length of the Interdigital Transducers (IDTs) for both structures were measured and compared with theoretical calculations. 
$\mathrm{ZnO} / 90^{\circ}$ rotated ST-cut quartz crystal structure shows electromechanical coupling coefficient as high as 0.06 due to the high piezoelectricity of $\mathrm{ZnO}$, making it suitable for fabrication of acoustic low insertion loss filters. The propagation modes show no coupling with other modes. Capacitance per length of finger pairs is comparable to that of the Rayleigh mode blank quartz crystal structures. High speed of propagation of Love mode acoustic waves in the structure makes the dimensions of the filters smaller than those with Rayleigh and SH-SAW modes. These advantages suggest that the Love mode structures show remarkable performance as SAW filters. Further investigation is needed to analyse the dispersion effect of dispersion of the acoustic waves.

\section{ACKNOWLEDGMENT}

The authors would like to thank Cooperative Research Centre (CRC) for Micro-Technology, Australia, for the financial support.

\section{REFERENCES}

[1] C. K. Campbell, Surface Acoustic Wave Devices for Mobile and Wireless Communications, Academic Press, 1998.

[2] A. A. Oliner, Topics in Applied Physics: Acoustic Surface Waves, Springer-Verlag, New York, vol. 24, 1978.

[3] G. W. Farnell, I. A. Cermak, P. Silvester, and S.K. Wong, "Capacitance and Field Distributions for Interdigital Surface-Wave Transducers," IEEE Trans. Sonics on Ultrason., vol. 20, pp. 188-195, 1970.

[4] K. Kalantar-zadeh, W. Wlodarski, and K. Galatsis, "Love Mode Layered Surface Acoustic Wave Filters," Electronics Letters, vol. 37, no. 12, pp. 802-803, 2001.

[5] U. Wolf, F. L. Dickert, G. K. Fischrauer, W. Greibl, and C. C. W. Ruppel, "SAW Sensors for Harsh Environments," IEEE Sensors Journal, vol. 1, no. 1, pp. 413, 2001.

[6] F. Herrmann, M. Weihnacht, and S. Buttgenbach, "Properties of Shear-Horizontal Surface Acoustic Waves in Different Layered Quartz-SiO ${ }_{2}$ Structures", Ultrasonics, vol. 37, pp. 335-341, 1999. 